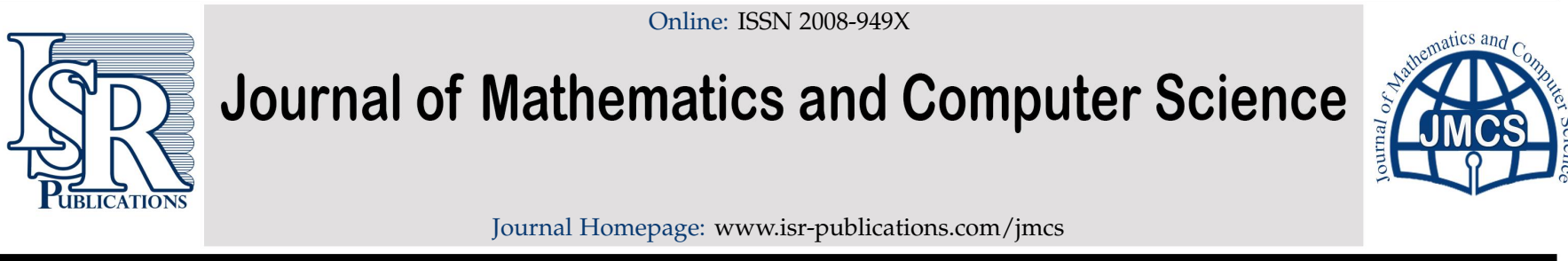

\title{
Semi-analytical solution for a system of competition with production a toxin in a chemostat
}

\author{
Aisha Abdullah Alderremy ${ }^{\mathrm{a}}$, Mourad Chamekh ${ }^{\mathrm{b}, \mathrm{d}, *}$, Fadhel Jeday $^{\mathrm{c}, \mathrm{d}}$ \\ ${ }^{a}$ Mathematics Department, College of Sciences, King Khalid University, KSA. \\ ${ }^{b}$ Mathematics Department, College of Sciences and Arts, AlKamel, University of Jeddah, KSA. \\ ${ }^{c}$ Mathematics Department, Jamoum College, Umm Al-Qura University, KSA. \\ ${ }^{d}$ National Engineering School at Tunis, University of Tunis EI Manar, LAMSIN, 1002, Tunis, Tunisia.
}

\begin{abstract}
The resolution of a system for modeling the competition between opponents in a chemostat when one of these can produce a toxin has been studied. We propose a novel method to overcome the analytical difficulties of standard mathematical methods. The method is based on the variational iteration method and combined with the Gauss-Seidel technique for increasing the convergence rate. Numerical examples are considered to demonstrate the practicality and improve the convergence of the proposed method.
\end{abstract}

Keywords: VIM, semi-analytical solution, nonlinear Gauss-Seidel method, opponents in a chemostat. 2010 MSC: 47E05, 35F30.

(C)2020 All rights reserved.

\section{Nomenclature}

$S$ concentrations of nutrient

$X$ concentrations of the toxin sensitive microorganism

$\mathrm{P}$ concentrations of toxin present

$\mathrm{Y}$ concentrations of toxin producing organism

\section{Introduction}

Following Hsu and Waltman in [11], a system for modeling the competition between opponents in a chemostat when one of these can produce a toxin is given by

$$
\frac{d}{d t} S=\left(S^{0}-S\right) D-\frac{m_{1} S}{\gamma_{1}\left(a_{1}+S\right)} X-\frac{m_{2} S}{\gamma_{2}\left(a_{2}+S\right)} Y,
$$

\footnotetext{
*Corresponding author

Email addresses: aaldramy@kku.edu.sa (Aisha Abdullah Alderremy), chamekhmourad1@gmail.com \& mourad.chamekh@enit.utm.tn (Mourad Chamekh), fadheldj@yahoo.com (Fadhel Jeday)

doi: 10.22436/jmcs.020.02.07
}

Received: 2019-04-09 Revised: 2019-06-21 Accepted: 2019-07-30 


$$
\begin{aligned}
\frac{d}{d t} X & =\left[\frac{m_{1} S}{\left(a_{1}+S\right)}-D-\gamma P\right] X, \\
\frac{d}{d t} Y & =\left[\frac{(1-K) m_{2} S}{\left(a_{2}+S\right)}-D\right] Y, \\
\frac{d}{d t} P & =\frac{K m_{2} S}{\left(a_{2}+S\right)} Y-D P,
\end{aligned}
$$

with the variables $S, X$, and $P$ represent at time $t$, the concentrations of nutrient in the vessel, of the toxin sensitive microorganism and of toxin present, respectively, and $Y$, the toxin producing organism. The parameter $S^{0}$ is the input nutrient concentration, $D$ is the washout rate, $m_{1,2}$ is the maximal growth rate, $a_{1,2}$ is the Michaelis-Menten constant, and $\gamma_{1,2}$ is the yield constant. The parameter $\mathrm{k}$ represents the fraction of potential growth devoted to producing the toxin. For more information on the mathematical modeling and analysis of subject, we can see for example the works of El Hajji et al. [5-7].

Using a procedure of normalization including an operation of reduction of variables of problem (1.2) can lead to the reduced form [11]

$$
\begin{aligned}
& \frac{d s}{d t}=1-s-\frac{m_{1} s}{a_{1}+s} x-\frac{m_{2} s}{a_{2}+s} y, \\
& \frac{d x}{d t}=\left[\frac{m_{1} s}{\left(a_{1}+s\right)}-1-\frac{k \gamma}{1-k} y\right] x, \\
& \frac{d y}{d t}=\left[\frac{(1-k) m_{2} s}{\left(a_{2}+s\right)}-1\right] y .
\end{aligned}
$$

A lot of semi-analytical methods have recently developed to solving nonlinear physical problems. Among those are, for example, the variation iteration method (VIM) [10], the Elzaki transform [8, 9], novel iteration method $[1-4,13]$ etc.. Although these methods have presented attracted great interest, this field may need further improvement.

In what follows in this paper, we shall propose some semi-analytical solutions for problem (1.2).

\section{Solution by variational iteration method}

The VIM is a real method invented by He [10] since 1999. Since then, this method has been intensively used in the literature of semi-analytical methods because of its broad applicability and efficiency for some difficult physical problems.

The principle of the method used is as follows.

We consider system (1.2) in the following integrals form:

$$
u_{n+1}(y)=u_{n}(y)+\int_{0}^{t} \lambda(\xi)\left(\tilde{u}^{\prime}(\xi)-\mathcal{L} \tilde{u}(\xi)-\mathcal{N} \tilde{u}(\xi)-g(\xi)\right) d \xi,
$$

with $u=(s, y, x), \lambda=\left(\lambda_{1}, \lambda_{2}, \lambda_{3}\right)$ being a vector containing the Lagrangian multipliers, and $L$ and $N$ are the linear and the nonlinear parts of system (1.2), respectively. Therefore,

$$
\begin{aligned}
& s^{n+1}(t)=s^{n}(t)+\int_{0}^{t} \lambda_{1}(\xi)\left(\left(s^{n}\right)^{\prime}-1+s^{n}+\frac{m_{1} \tilde{s}^{n}}{a_{1}+\tilde{s}^{n}} \tilde{x}^{n}+\frac{m_{2} \tilde{s}^{n}}{a_{2}+\tilde{s}^{n}} \tilde{y}^{n}\right) d \xi \\
& y^{n+1}(t)=y^{n}(t)+\int_{0}^{t} \lambda_{2}(\xi)\left[\left(y^{n}\right)^{\prime}-\left(\frac{(1-k) m_{2} \tilde{s}^{n+1}}{\left(a_{2}+\tilde{s}^{n+1}\right)}\right) \tilde{y}^{n}-y^{n}\right] d \xi \\
& x^{n+1}(t)=x^{n}(t)+\int_{0}^{t} \lambda_{3}(\xi)\left[\left(x^{n}\right)^{\prime}-\left(\frac{m_{1} \tilde{s}^{n+1}}{\left(a_{1}+\tilde{s}^{n+1}\right)}-\frac{k \gamma}{1-k} \tilde{y}^{n+1}\right) \tilde{x}^{n}-x^{n}\right] d \xi .
\end{aligned}
$$

This proposed algorithm is exceptionally based on the Gauss-Seidel technique developed by Friedrich Gauss and Philipp Ludwig von Seidel. Compared with the classical VIM, where the solution obtained in the $n$-th iteration remains fixed until the entire $n+1$-th iteration has been realized. As in (2.1a) and (2.1b), we used the new values as soon as they are known. 
Using $\delta \tilde{u}(\xi)=0$, we obtain the following optimal value of the Lagrangian multipliers

$$
\lambda=-\left(e^{\xi-t}, e^{\xi-t}, e^{\xi-t}\right) .
$$

The modified variational iterations algorithm is given by:

$$
\begin{aligned}
s^{0} & =s(0), y^{0}=y(0), \text { and } x^{0}=x(0), \\
s^{n+1}(t) & =s^{n}(t)-\int_{0}^{t} e^{\xi-t}\left[\left(s^{n}\right)^{\prime}-1+s^{n}+\frac{m_{1} s^{n}}{a_{1}+s^{n}} x^{n}+\frac{m_{2} s^{n}}{a_{2}+s^{n}} y^{n}\right] d \xi, \\
y^{n+1}(t) & =y^{n}(t)-\int_{0}^{t} e^{\xi-t}\left[\left(y^{n}\right)^{\prime}-\left(\frac{(1-k) m_{2} s^{n+1}}{\left(a_{2}+s^{n+1}\right)}-1\right) y^{n}\right] d \xi, \\
x^{n+1}(t) & =x^{n}(t)-\int_{0}^{t} e^{\xi-t}\left[\left(x^{n}\right)^{\prime}-\left(\frac{m_{1} s^{n+1}}{\left(a_{1}+s^{n+1}\right)}-\frac{k \gamma}{1-k} y^{n+1}-1\right) x^{n}\right] d \xi,
\end{aligned}
$$

with $s(0), y(0)$, and $x(0)$ represent the initial conditions of problem. However, we note that the classical VIM leads to the following algorithm

$$
\begin{aligned}
s^{0} & =s(0), y^{0}=y(0), \text { and } x^{0}=x(0), \\
s^{n+1}(t) & =s^{n}(t)-\int_{0}^{t} e^{\xi-t}\left[\left(s^{n}\right)^{\prime}-1+s^{n}+\frac{m_{1} s^{n}}{a_{1}+s^{n}} x^{n}+\frac{m_{2} s^{n}}{a_{2}+s^{n}} y^{n}\right] d \xi, \\
y^{n+1}(t) & =y^{n}(t)-\int_{0}^{t} e^{\xi-t}\left[\left(y^{n}\right)^{\prime}-\left(\frac{(1-k) m_{2} s^{n}}{\left(a_{2}+s^{n}\right)}-1\right) y^{n}\right] d \xi, \\
x^{n+1}(t) & =x^{n}(t)-\int_{0}^{t} e^{\xi-t}\left[\left(x^{n}\right)^{\prime}-\left(\frac{m_{1} s^{n}}{\left(a_{1}+s^{n}\right)}-\frac{k \gamma}{1-k} y^{n}-1\right) x^{n}\right] d \xi .
\end{aligned}
$$

\section{Numerical examples}

In this first example we have chosen the following parameter values

$$
a_{1}=0.2, a_{2}=3, m_{1}=1, m 2=5, k=0.3 \text {, and } G=1 .
$$

According to the results of $[11,12]$, we have an equilibrium point $E_{0}=(1,0,0)$ always exists and it is globally asymptotically stable if $\frac{m_{1}}{\left(a_{1}+1\right)}<1$ and $\frac{m_{2}}{\left(a_{2}+1\right)}<(1-k)^{-1}$. Thanks to these conditions, we have indeed a globally asymptotically stability, since $\frac{m_{1}}{\left(a_{1}+1\right)}=0.8333$ and $\frac{m_{2}}{\left(a_{2}+1\right)}=1.2500$ and $(1-\mathrm{k})^{-1}=1.4286$.

Basing of the classical VIM (2.3), the semi-analytical solutions of $S, Y$, and $X$ are given by

$$
\begin{aligned}
& S_{0}=S(0)=1, Y_{0}=Y(0)=\frac{3}{10}, X_{0}=X(0)=\frac{7}{10}, \\
& S_{1}=\frac{1}{24}\left(e^{-t}+1\right), Y_{1}=\frac{1}{80}\left(3 e^{-t}+21\right), X_{1}=\frac{1}{150}\left(31 e^{-t}+74\right), \\
& S_{2}=-e^{-t}\left[\frac{1142}{553} t+\frac{181}{125} \log \left(115 e^{-t}+29\right)+\frac{475}{2131} \log \left(\frac{23 e^{-t}+73}{96}\right)-\frac{362}{125} \log (12)-\frac{897}{1000} e^{t}-\frac{103}{1000}\right], \\
& Y_{2}=e^{-t}\left[\frac{181}{630} t+\frac{615}{3941} \log \left(\left(23 * e^{-t}+73\right) / 96\right)+\frac{147}{11680} e^{t}+\frac{797}{2773}\right], \\
& X_{2}=\frac{124}{1250}+\left[\frac{513}{769}+\frac{181}{125} \log \left(\frac{115}{144} e^{-t}+\frac{29}{144}\right)+\frac{677}{417} t\right] e^{-t}+33.10^{-4} e^{-2 t} .
\end{aligned}
$$

However, these solutions can be achieved with using the modified VIM (2.2),

$$
S_{0}=S(0)=1, Y_{0}=Y(0)=\frac{3}{10}, X_{0}=X(0)=\frac{7}{10},
$$




$$
\begin{aligned}
& S_{1}=\frac{1}{24}\left(e^{-t}+1\right), \\
& Y_{1}=e^{-t}\left[\frac{803 t}{2461}-45.10^{-4} \log \left(23 e^{-t}+73\right)+\frac{483}{1460} \log \left(\frac{23 e^{-t}+73}{96}\right)+\frac{21 e^{t}}{1460}+\frac{271}{7078}\right], \\
& X_{1}=\frac{1}{150}\left(31 e^{-t}+74\right) .
\end{aligned}
$$

\section{Results and discussions}

In order to evaluate the VIM approximation, we have proposed to compare the solutions of this method with theirs obtained by fourth-order Runge-Kutta procedures (RK4). This choice is justified by the fact that it is not easy to calculate a closed form solution but also that the RK4 method is very efficient and precise. For this reason, we have used RK4 solutions as exact solutions, we have therefore calculated the relative errors on this basis.

When $t \in[01]$, we have a relative maximum error does not exceed $4 \times 10^{-3}$ for the all approximate solutions of $S, Y$ and $X$. The obtained solutions give an interesting view of the efficiency of the VIM used. With just past two iterations we can remark that the error is negligible, shown in Fig. 3 and explained in Table 1. Fig. 2 shows the comparison between traditional and modified VIM solutions in one hand and Range Kutta (RK4) numerical solutions in the other hand in a relatively wide time span. We noted that the branches of the solution begin to be improved especially with the $X$ solution, Fig. 2.

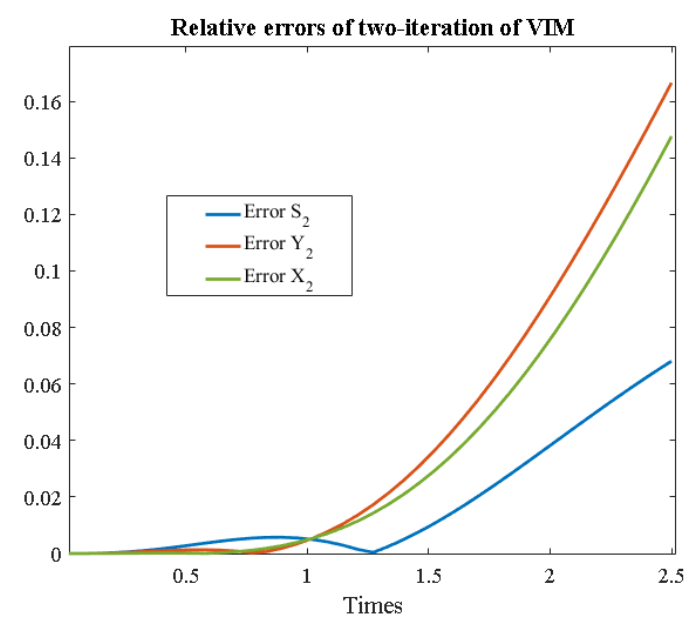

Figure 1: The relative errors of modified VIM solutions.

Table 1: Summarized of relative errors.

\begin{tabular}{|c|c|}
\hline $\mathrm{t}$ & VIM relative error \\
\hline 0 & 0 \\
0.1048 & $8 \times 10^{-6}$ \\
0.2096 & $5 \times 10^{-5}$ \\
0.2721 & $9 \times 10^{-5}$ \\
0.3971 & $1.5 \times 10^{-4}$ \\
0.4596 & $1.5 \times 10^{-4}$ \\
0.5846 & $3 \times 10^{-5}$ \\
0.7096 & $6.2 \times 10^{-4}$ \\
0.8346 & $1.8 \times 10^{-3}$ \\
0.9596 & $3.9 \times 10^{-3}$ \\
\hline
\end{tabular}



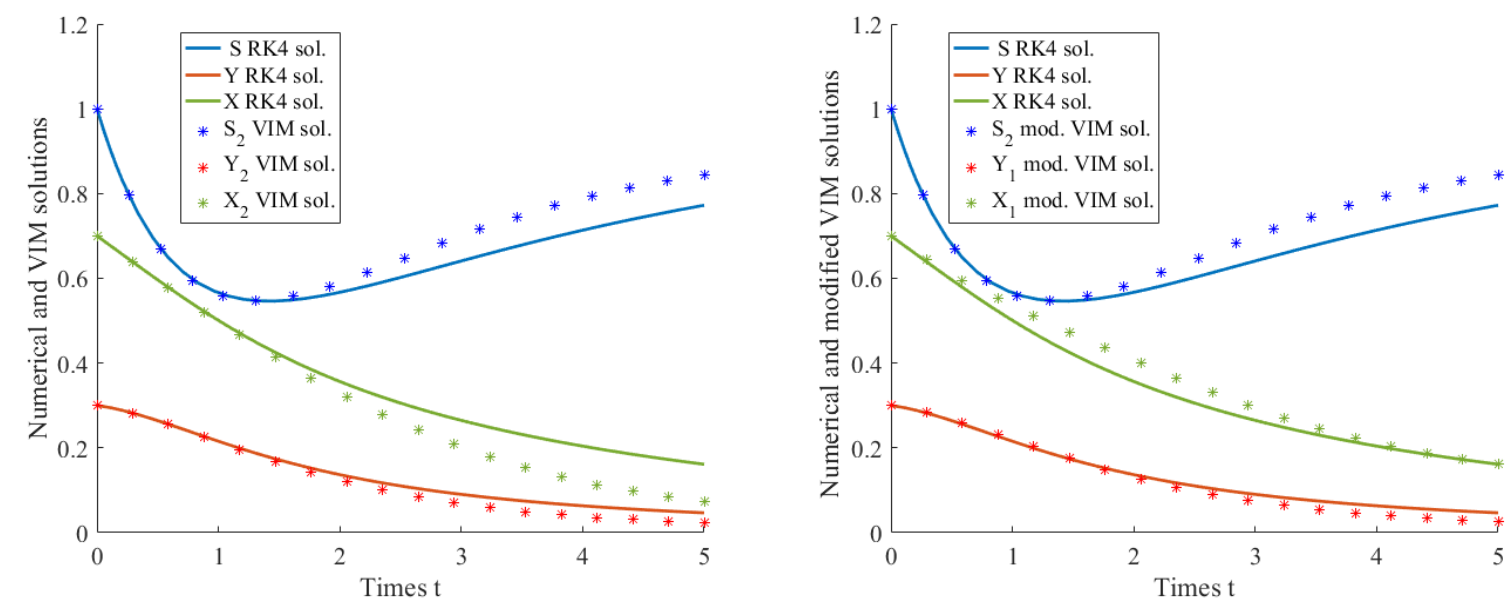

Figure 2: We present the comparison between the classical VIM solutions and the numerical Range Kutta (RK4) solutions within a relatively broad time interval (left figure). In the right, we compare the modified VIM solutions with RK4 solutions (right figure).

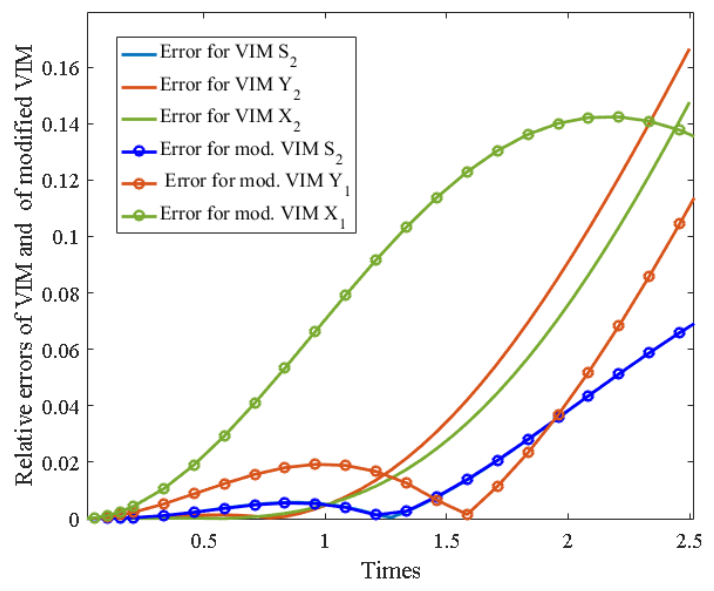

Figure 3: Comparison between the relative error of modified VIM and VIM solutions.

\section{Conclusion}

We have proposed a modified VIM solution for a system of competition with production a toxin in a chemostat. In conjunction with other methods, VIM adapts rapidly and flexibly to the Gauss-Seidel technique. Thanks to this combining, we have highlighted the efficiency of this modification to improve the convergence of solutions.

\section{Acknowledgment}

The authors extend their appreciation to the Deanship of Scientific Research at King Khalid University for funding this work through Research Groups Program under Grant no. (R.G.P.1 /64 /40).

\section{References}

[1] G. Adomian, Convergent series solution of nonlinear equation, J. Comput. Appl. Math., 11 (1984), 113-117. 1

[2] A. A. Alderremy, T. M. Elzaki, M. Chamekh, New transform iterative method for solving some Klein-Gordon equations, Results Phys., 10 (2018), 655-659. 
[3] A. A. Alderremy, T. M. Elzaki, M. Chamekh, Modified Adomian Decomposition Method to Solve Generalized EmdenFowler Systems for Singular IVP, Math. Probl. Eng., 2019 (2019), 6 pages.

[4] M. Chamekh, T. M. Elzaki, N. Brik, Semi-analytical solution for some proportional delay diferential equations, SN Appl. Sci., 148 (2019), 6 pages. 1

[5] M. El Hajji, How can inter-specific interferences explain coexistence or confirm the competitive exclusion principle in a chemostat, Int. J. Biomath., 11 (2018), 20 pages. 1

[6] M. El Hajji, N. Chorfi, M. Jleli, Mathematical model for a membrane bioreactor process, Electron. J. Differential Equations, 2015 (2015), 7 pages.

[7] M. El Hajji, N. Chorfi, M. Jleli, Mathematical modelling and analysis for a three-tiered microbial food web in a chemostat, Electron. J. Differential Equations, 2017 (2017), 13 pages. 1

[8] T. M. Elzaki, A solution of nonlinear diferential equations using mixture of Elzaki transform and transform method, Int. Math. Forum, 13 (2012), 631-638. 1

[9] T. M. Elzaki, M. Chamekh, Solving nonlinear fractional differential equations using a new decomposition method, Univ. J. Appl. Math. Comput., 6 (2018), 27-35. 1

[10] J. H. He, Variational iteration method a kind of nonlinear analytical technique: some examples, Int. J. Nonlinear Mech., 34 (1999), 699-708. 1, 2

[11] S. B. Hsu, P. Waltman, Competition in the chemostat when one competitor produces a toxin, Japan J. Indust. Appl. Math., 15 (1998), 471-790. 1, 1, 3

[12] H. R. Thieme, Persistence under relaxed point-dissipativity (with application to an epidemic model), SIAM J. Math. Anal., 24 (1992), 407-435. 3

[13] J. K. Zhou, Differential transformation and its application for electrical circuit (in Chinese), Huazhong University Press, Wuuhahn, (1986). 1 